\title{
Torri difensive e territori fluviali: architetture d'acque nel bacino del fiume Zhujiang, Guangdong
}

Defensive towers and river territories: water architecture in the Zhujiang river basin, Guangdong

\author{
Loredana Ficarelli $^{\text {a }}$, Valentina Vacca ${ }^{\text {b }}$ \\ a Dipartimento di Scienze dell'Ingegneria Civile e dell'Architettura - Politecnico di Bari, Bari, Italy, \\ loredana.ficarelli@poliba.it

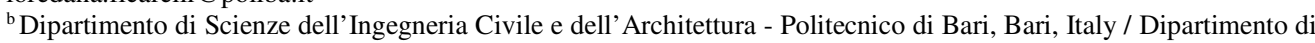 \\ Architettura - Università degli Studi Roma Tre, Rome, Italy, valentina.vacca@poliba.it
}

\begin{abstract}
The contribution intends to provide a reading and an in-depth study of the defensive heritage located in the Zhujiang river basin and its delta in Guangdong, China. The paper focuses on the case of diaolou, defensive towers already listed as UNESCO since 2007, built from the sixteenth century until the first half of the twentieth century in Kaiping country. These buildings show an interesting mixture of some local models and typologies and specific characters and styles borrowed from western examples.

The research takes as a privileged point of view the relationships that these settlement systems forge with the hydrographic resource, which generates a territorial groove that determines the morphology of the territory and constitutes a historical vehicle of crossings. The arrangement of the fortified towers with respect to the river line is influenced by centripetal and centrifugal actions aimed at responding to defensive needs in the geography of this territory.

The heritage of the diaolou seems to respond to two types of defensive demands: one linked to historical facts and the frequent bandit raids that took place in the Guangdong area in the nineteenth century; the other connected to geographical and hydraulic data, as the protection from the phenomenon of inundation and the consequent placement of the towers in the floodplain of the Zhujiang river. The course of the river gets in shape through the architectural technique, in the construction of towers and defensive works and, in the same way, some aspects of the design of this territory are defined through the description of the forms of the river.

Architecture, hydraulic engineering and geography work together in defining the form of the settlement and invest the scale of the buildings, generating specific architectural types and morphological characters suitable for responding to the problem of water control, conservation and distribution.
\end{abstract}

Keywords: Diaolou, chinese towers, river, floodplain.

\section{Presupposti teorici della ricerca}

La ricerca si inserisce in un ambito più ampio di studi che si pongono l'obiettivo di ampliare il bagaglio delle analisi sulla città e sul territorio, al fine di contribuire al processo progettuale, attraverso una rinnovata attenzione ai fatti geografici e alla forma del suolo.
"Di fronte alla dispersione attuale della città, alla caduta dei limiti che disegnandola e circoscrivendola anche formalmente la contrapponevano alla campagna e a maggior ragione ai luoghi incolti, l'attenzione ai fatti geografici che l'attraversano e in base ai quali si può ripensare al disegno delle diverse forme insediative" (Motta, et al., 2006, p. 9) induce a ripartire dalla "in- 
trinseca architettonicità" (Motta, et al., 2006, p. 10) di elementi naturali come rilievi montuosi, sistemi di colline, pianure, fiumi, isole o porzioni di costa. Nella relazione con gli elementi della natura, l'architettura raggiunge il massimo grado di astrazione, si distacca dal disegno urbano e diviene archetipo; nell'archetipo il dato naturale si trasforma in fatto costruito e quindi si manifesta come terra emersa o piramide, basamento e recinto, arca e tempio, camino, ponte, ciminiera e diga, oppure torre (Motta, et al., 2006, pp. 1920).

A partire da questo filone di ricerca, il contributo osserva e indaga le relazioni tra i sistemi difensivi a torre e i tracciati idrografici, intesi come solchi territoriali che articolano la morfologia del suolo e veicolano costantemente attraversamenti e flussi. La torre corrisponde all'archetipo che affiora spontaneamente rispetto alle terre emerse e alle acque. Attraverso di essa è possibile traguardare il territorio e alterare il consueto rapporto con la linea di orizzonte. La sua forma interferisce con il profilo morfologico del territorio e stabilisce relazioni visive con parti di esso anche a lunga distanza. Per sua definizione, la torre come archetipo si traduce in forma nelle opere difensive, elementi puntuali a servizio delle necessità di controllo del territorio; la sua forma evoca e cristallizza quella delle guglie delle montagne. Come le alture emergono dalle terre emerse, così le torri emergono dal profilo del fiume, nuovi picchi costruiti dall'uomo, nuove architetture della terra (Motta, et al., 2006, p. 21).

\section{Metodologia d'indagine}

La ricerca si avvale del contributo di diverse discipline, a servizio del tema più vasto dello studio del territorio.

In prima analisi, si è fatto ricorso agli strumenti delle discipline geografiche e idrografiche, che chiariscono le configurazioni d'alveo di un fiume, spiegando i fattori naturali che agiscono come vocazioni insediative delle porzioni del territorio; questi studi consentono anche di descrivere $\mathrm{i}$ cambiamenti a cui sono soggetti i corsi d'acqua, le variazioni nella geometria del loro tracciato, i progressivi adattamenti indotti dalle trasformazioni del territorio su cui i corsi d'acqua insistono. Lo studio della morfologia del fiume permette anche l'individuazione di alcuni punti notevoli che si costituiscono come condizioni di sito per l'insediamento e ne deter- minano la forma; tra queste condizioni figurano: la sorgente, i meandri e le sponde concave e convesse, i luoghi in cui l'alveo si dipana in rami paralleli, le confluenze tra due corsi d'acqua, le isole e gli istmi, il delta e la foce (Motta, Ravagnati, 2008, p. 111).

In parallelo, la ricerca è stata condotta anche attraverso la comprensione della struttura degli insediamenti e lo studio tipologico degli edifici a torre, intendendo per tipo architettonico "un enunciato che descrive una struttura formale" $\mathrm{e}$ quindi astraendo una descrizione degli edifici "di natura concettuale, non oggettuale" (Martí Arís, 1994, p. 16).

L'obiettivo della ricerca è di elaborare nuove categorie utili all'interpretazione dei paesaggi fluviali come fatti architettonici; questa lettura intende osservare le relazioni di forma tra i sistemi difensivi a torre e l'idrografia, interpretando questo patrimonio insediativo come opportunità per progettare il fiume nel territorio contemporaneo.

\section{Ambito geografico di studio: il delta del fiume Zhujiang e la contea di Kaiping}

L'ambito di studio individuato per la ricerca è il bacino del fiume Zhujiang, in prossimità del suo delta in Guangdong. La rete fluviale capillare, che da millenni solca questo territorio, ha determinato in modo quasi esclusivo la sua morfologia con i suoi processi di erosione e di lento deposito di sedimenti e ha reso questo territorio storicamente permeabile a flussi diversificati, migrazioni e influenze colonialiste.

La scelta dell'ambito di studio è riconducibile alle recenti opportunità di indagine accolte dal gruppo di ricerca presso la Scuola di Architettura del Politecnico di Bari ${ }^{1}$ e all'interesse nei confronti del contesto geografico cinese per le sue trasformazioni in atto.

Nel territorio del delta del fiume Zhujiang si riconosce, inoltre, la rilevanza della rete fluviale nello studio dei tipi e dei caratteri difensivi, poiché in questo luogo molti di essi si manifestano in presenza del solco idrografico. Questo carattere emerge in modo significativo nelle rappresentazioni cartografiche della città di Guangzhou, che mostrano le relazioni che il sistema murario della città stabilisce con il tracciato idrografico, fortificando i suoi varchi in prossimità della linea del fiume e raddoppiando la cinta muraria seguendo il margine delle terre emerse. 


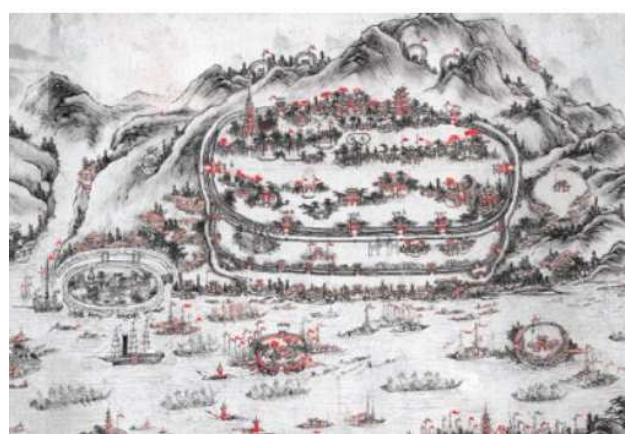

Fig. 1. Mappa di Guangzhou del 1892 (Bosselmann, 2018).

A sinistra della città murata, la mappa mostra anche l'isola fortificata di Shameen, che figura tra i porti dei trattati ineguali concessi per il libero commercio alle potenze occidentali con il trattato di Nanchino (1842). Un canale artificiale separa l'isola dalla terra ferma, confermando l'utilizzo dei corsi d'acqua come elementi cruciali per l'assetto difensivo del delta; in questo modo l'isola mantiene un rilevante grado di autonomia rispetto al tessuto della città di Guangzhou, che conserva ancora oggi.

A circa 110 km di distanza dalla città di Guangzhou, nella parte ovest del delta del fiume Zhujiang, si situa la contea di Kaiping, collocata nella zona centro-meridionale del Guangdong (Tan, 2013, p. 1). La contea è attraversata da ovest a est dal fiume Tanjiang e la forma del suo territorio è piuttosto variegata, con il solco idrografico circondato da aree collinari e pianeggianti nella parte centro-orientale e rilievi montuosi nelle altre aree; le parti pianeggianti con altitudine inferiore ai $50 \mathrm{~m}$ costituiscono il $69 \%$ della superficie territoriale della contea, mentre il restante 29\% della superficie è collinare (Wang, 2016, p. 12).

In questa contea si collocano i villaggi, già tutelati da UNESCO dal 2007, che ospitano numerosi esempi di architetture fortificate singolari, denominate diaolou (碉楼), complessi di torri multipiano con funzioni difensive, costruiti a partire dalla fine del periodo della Dinastia Ming (13681644) nella contea. La diffusione capillare di questi edifici, tuttavia, è relativa al periodo compreso tra il XIX secolo e la prima metà del XX secolo, in cui la regione del Guangdong era interessata da disordini sociali e incursioni straniere.

A questo proposito, Selia Tan che si è occupata diffusamente dei diaolou, contribuendo al loro censimento e alla candidatura UNESCO, chiarisce che "Tre fattori spiegano la funzionalità dei diaolou e contribuiscono alla loro diffusione: primo, allo scopo di difendere. L'ordine sociale di Kaiping era scarso e i banditi erano incontrollabili. Secondo, allo scopo di (proteggere dalle $n d r$ ) inondazioni. Kaiping era una valle circondata da montagne e fiumi, quindi le inondazioni erano comuni durante la stagione dei monsoni. Gli abitanti del luogo avrebbero costruito torri di controllo per proteggere loro stessi dai banditi e dalle inondazioni. Terzo, al fine di abitare" (Tan, 2007, pp. 16-17). Ai fini dello studio, quindi, si intende rimarcare i primi due fattori e fornire un'interpretazione del patrimonio difensivo dei diaolou come tipologia a torre che risponde a due possibili istanze difensive: una legata ai fatti storici e alle frequenti incursioni banditesche, l'altra più strutturale, connessa al dato geografico e idrografico.

Da un punto di vista storico, i disordini sociali e le frequenti migrazioni sono documentate in questa area del Guangdong, già a partire dal periodo della Dinastia Ming; a seguito dell'istituzione di Kaiping come contea nel 1649 , quest'area attraversò un periodo di relativa pace che si concluse a metà del XIX secolo. La guerra dell'Oppio (1840-1842), l'insurrezione di Taiping (1850-1864), la "rivolta dell'esercito della sciarpa rossa" (1854), l'imperialismo straniero e i conflitti armati a carattere etnico tra la popolazione locale e i coloni Hakka furono tra le principali cause di disordini. Il Trattato di Nanchino (1842), il Trattato di Pechino (1860) e il Trattato di Burlingame (1868) concessero a Gran Bretagna, Francia e America la possibilità di disporre di porti dei trattati ineguali in Cina e facilitarono la recluta di lavoratori cinesi disposti a lavorare all'estero; questo fenomeno si diffuse in modo significativo nell'area di Kaiping e coinvolse centinaia di migliaia di cittadini dell'area che si trasferirono in Nord America, America Latina, nel sud-est asiatico e nell'area del Pacifico. Incapaci di stabilirsi in questi Paesi, anche a causa di frequenti discriminazioni, molti di loro programmarono con cura il ritorno nei loro luoghi nativi, in preda a nuove ondate di brigantaggio causate dall'inefficacia dei governi locali. Al loro rientro, finanziarono e costruirono nuovi diaolou sempre più sofisticati, avvalendosi delle esperienze e delle conoscenze scientifiche maturate a contatto con la cultura occidentale (Tan, 2013, pp. 1-2). 


\section{Il fiume: elemento determinante per il si- stema difensivo.}

Il paesaggio del delta del fiume Zhujiang ha instaurato una relazione diversificata e articolata con le acque fluviali che include due principali categorie di risorse: la linea del fiume e gli stagni, legati al feng shui dei villaggi e alla produzione alimentare e ittica.

Geomorfologicamente, il delta del fiume si compone di tre sotto-delta, afferenti rispettivamente al fiume Xijiang (ramo occidentale), al fiume Beijiang (ramo settentrionale) e al fiume Dongjiang (ramo orientale). Beneficiando delle fertili pianure alluvionali e delle tecniche agricole avanzate, negli ultimi mille anni il fiume Zhujiang ha determinato un grande sviluppo di tutto il territorio del delta (Zhang, et al., 2008, p. 2224).

A questo proposito, Weng spiega diffusamente le principali strategie di gestione dei bacini idrografici attuate nel delta del fiume Zhujiang e chiarisce che le risorse idriche sono state il fattore più importante che ha influenzato il processo di civilizzazione di questo territorio negli ultimi due millenni. Lo stesso suolo si è originato principalmente a valle di lunghi processi di deposito di fertili sedimenti alluvionali, grazie all'azione dei tre principali rami del fiume e dei numerosi affluenti e distributori in questa zona (Weng, 2007, p. 1061).

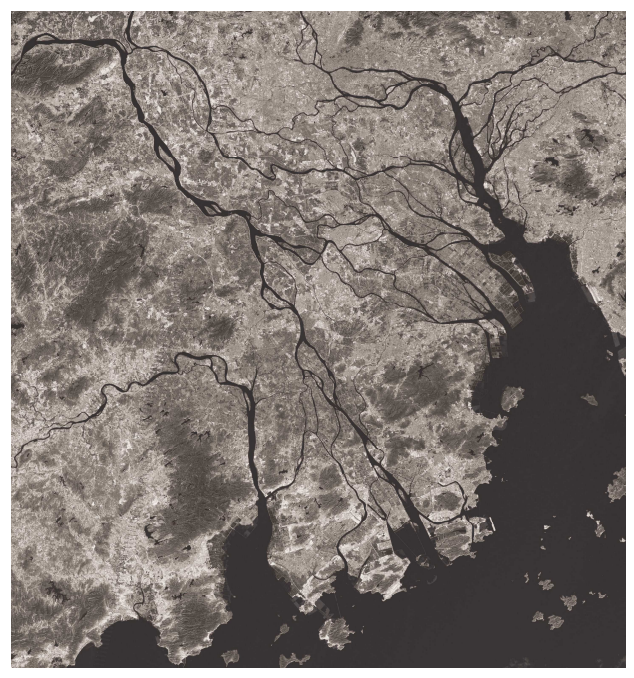

Fig. 2. Mappa del delta del fiume Zhujiang (Elaborazione a cura di Vacca V.).
Tuttavia, il fiume non è solo una risorsa per il territorio ma anche una minaccia; infatti, il delta del fiume Zhujiang è anche una regione storicamente vulnerabile ai danni delle alluvioni. Le cronache hanno registrato nel secolo scorso 45 gravi inondazioni in questa regione, di cui 36 tra il 1900 e nel 1949. L'alluvione del 1915, con periodo di ritorno di 200 anni, fu la peggiore mai registrata in molte località; causò danni ingenti ad oltre 935000 ha di terreni agricoli e colpì 100 mila abitanti, tra morti e feriti (Zhang, et al., 2008, p. 2224).

I processi trasformativi che interessano la piana inondabile del fiume hanno quindi sempre determinato delle condizioni di instabilità nel territorio in cui i diaolou si collocano, aggravate anche dalla collocazione di Kaiping in una fascia climatica monsonica subtropicale, in cui le precipitazioni sono molto frequenti e abbondanti. Gli eventi naturali sottopongono l'area a continue trasformazioni del suolo in cui si insediano i villaggi, periodicamente alterato nella sua forma dai processi erosivi e di deposito del fiume. Rispetto a queste condizioni, pertanto, emerge la necessità di difendersi dal fenomeno inondativo ricorrendo all'architettura, pianificando grandi vasche per la raccolta delle acque piovane e per la laminazione delle acque del fiume -vale a dire gli stagni già menzionati- e costruendo edifici a torre, i diaolou, in grado di rispondere alle necessità di difesa ergendosi rispetto alla condizione paludosa del territorio.

\section{Tipi di diaolou e loro collocazione a servizio dell'assetto difensivo}

Partendo dalle caratteristiche funzionali, il dossier elaborato per la candidatura Unesco suddivide i diaolou di Kaiping in tre categorie: torri residenziali, torri comunali e torri di avvistamento (The State Administration of Cultural Heritage of the People's Republic of China, 2007, p. 22).

A queste categorie funzionali spesso corrispondono anche diverse declinazioni del tipo, che rispondono con la forma dell'architettura alle differenti condizioni del sito. Il pattern difensivo della regione può quindi essere chiarito in modo esaustivo ricorrendo allo studio della forma dei diaolou, alla loro collocazione e ai conseguenti propositi difensivi. 

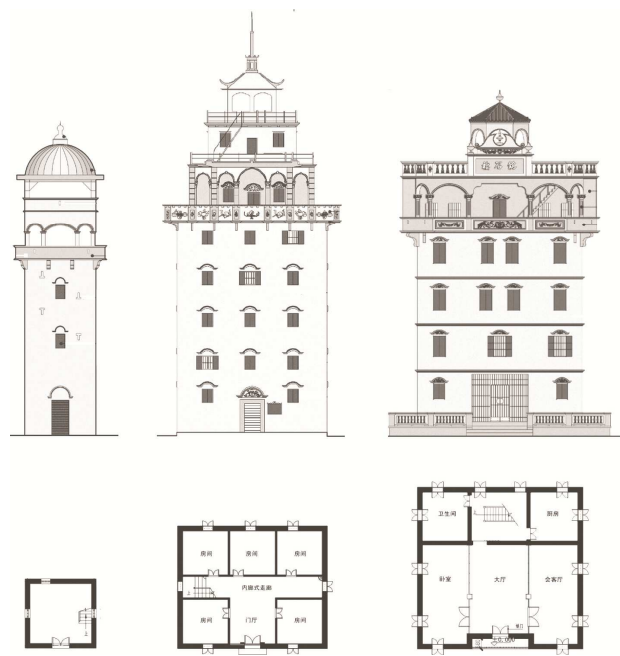

Fig. 3. Da sinistra: torri di avvistamento, torri comunali e torri residenziali a confronto (Elaborazione degli autori su Zhang, et al., 2012).

Le torri residenziali si insediano generalmente nella parte posteriore dei villaggi, per la volontà di un singolo proprietario. Questa categoria risponde in maniera combinata alle esigenze difensive e a quelle abitative (The State Administration of Cultural..., 2007, p. 22) e, pertanto, si manifesta spesso in tipi complessi, con un impianto generalmente tripartito, che deriva dalla tipologia abitativa più diffusa dell' area, chiamata three bay-two corridor ("tre vani, due corridoi" ndr). Gli elementi decorativi e la plastica secondaria in questi edifici vengono usati in abbondanza e con una certa libertà compositiva, che si avvale di riferimenti all'architettura occidentale. Le torri residenziali sono la categoria più diffusa di diaolou e rispondono alle necessità difensive del singolo nucleo famigliare.

Le torri comunali si collocano usualmente nella parte posteriore degli insediamenti e la loro costruzione era finanziata dalle famiglie del villaggio al fine di garantire un rifugio comune temporaneo in caso di incursioni armate o di inondazioni. Queste torri mostrano un chiaro assetto difensivo e lasciano spazio a pochi elementi decorativi (The State Administration of Cultural..., 2007, p. 22). Le torri comunali da un punto di vista tipologico si articolano come unità abitative collettive, con uno spazio distributivo comune trasversale all'ingresso e una successione di cellule lungo il perimetro. Sono le più antiche tra le tre categorie e partecipano al sistema difensi- vo legato al controllo territoriale del singolo villaggio.

Le torri di avvistamento si situano principalmente agli ingressi dei villaggi o in prossimità di luoghi strategici rispetto ai percorsi territoriali e alla linea del fiume ${ }^{5}$. In alcuni casi si collocano sui punti più alti dei rilievi circostanti. Da un punto di vista tipologico sono molto semplici e si configurano come semplici unità monocellulari che si reiterano verticalmente. L'appartenenza al tipo si manifesta anche nelle proporzioni di questi edifici sono generalmente molto slanciati, al fine di guadagnare la vista di ampie porzioni del territorio circostante. La collocazione di questo tipo di diaolou è pianificata in modo da presidiare il territorio, sfruttando le relazioni visive tra elementi puntuali; i luoghi privilegiati per l'insediamento di queste tipologie di torri sono quindi i punti più alti della piana inondabile o le zone adiacenti alle principali vie di comunicazione, tra le quali il fiume assume un ruolo di fondamentale importanza.

A titolo di esempio, la torre di avvistamento del clan Fang, si erge a sud del villaggio di Zili in cima a una collina. La costruzione risale al 1920 e fu finanziata dalla popolazione del villaggio di Zili e da altri villaggi nelle vicinanze dello stesso clan Fang. La torre, rivolgendosi a nord est, conquista un'altezza privilegiata per il controllo del territorio circostante e traguarda a ovest il percorso principale di accesso ai villaggi.

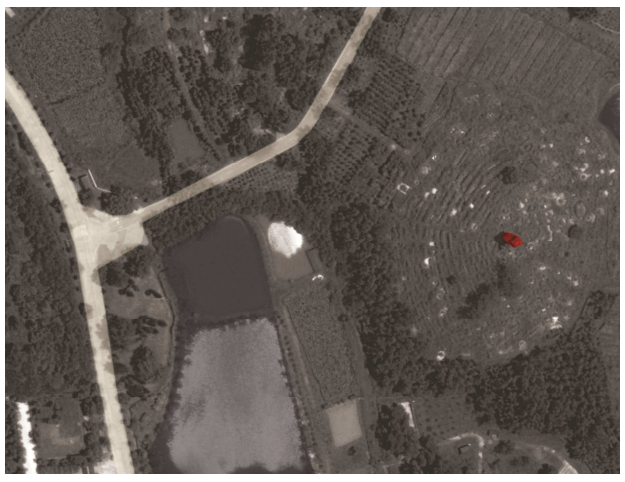

Fig. 4. Collocazione della torre di avvistamento del Clan Fang (Elaborazione a cura di Vacca V.).

L'altezza dell'edificio, le scarse aperture ai piani inferiori, serrate da persiane robuste, figurano tra i caratteri difensivi di questo diaolou. La veranda a coronamento della torre si conclude con un 
tetto a padiglione, coniugando le istanze difensive con la necessità di inserirsi nel paesaggio.

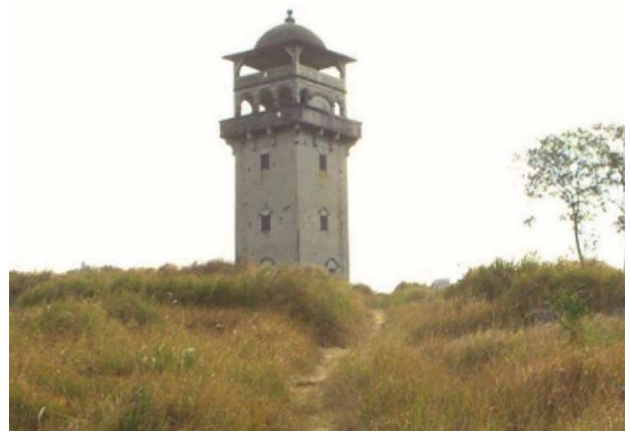

Fig. 5. Torre di avvistamento del Clan Fang (Yie, et al., 2019).

La torre di avvistamento denominata Nan Lou rappresenta un caso esemplificativo delle istanze difensive connesse al fiume. L'edificio si colloca in corrispondenza di un punto rilevante nella geografia del fiume Tanjiang, dove esso si ramifica in tre canali paralleli, solcando con il suo corso meandriforme la piana inondabile.

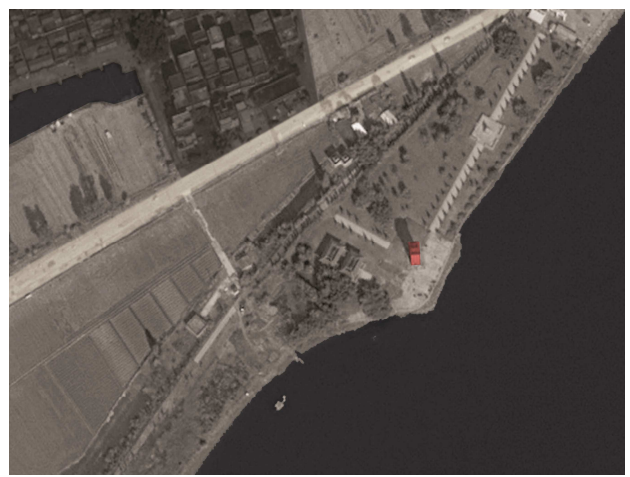

Fig. 6. Collocazione della torre di avvistamento Nan Lour (Elaborazione a cura di Vacca V.).

La sua collocazione presidia in modo efficace il corso del fiume e si avvale di un lieve terrazzo morfologico che contribuisce a sopraelevare l'edificio. Proseguendo il suo corso, poco più a ovest il fiume si piega in un ampio meandro, completamente inquadrato dalle piccole aperture disposte lungo i muri della torre.

In conclusione, la distribuzione di diaolou nell'area di Kaiping varia significativamente in base ai tre tipi di torri individuati. Le torri comunali e le torri residenziali seguono una distri- buzione sovrapponibile a quella dei villaggi, a sua volta influenzata dai fiumi e dalla topografia del territorio; queste torri e i loro villaggi di riferimento sono principalmente distribuiti nella piana centrale della contea, lungo un buffer del fiume Tanjiang, oppure a nord e sud di Kaiping in corrispondenza delle zone collinari. Le torri di avvistamento, invece, sono relativamente disperse nel territorio e contribuiscono ad un assetto difensivo policentrico, in grado di sovrintendere al territorio di tutta la contea (Liang, et al., 2017).

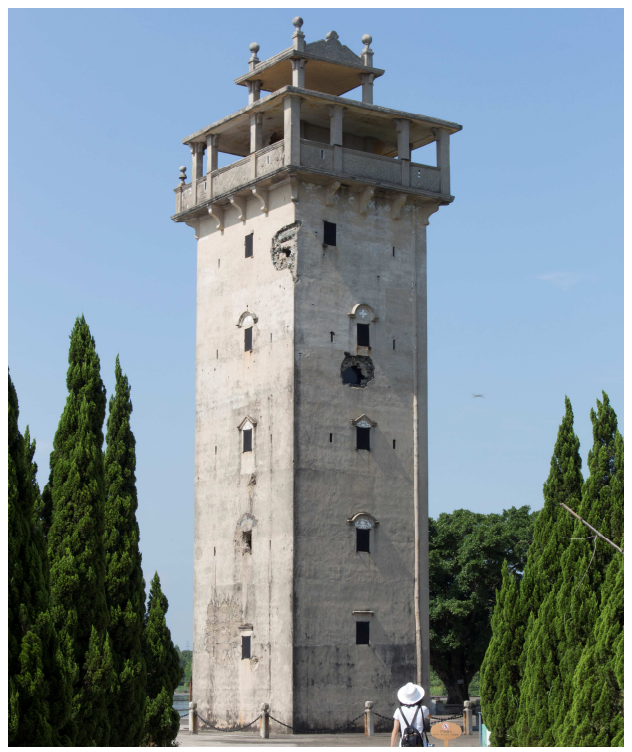

Fig. 7. La torre di avvistamento Nan Lour (Immagine Vacca V., 2018).

\section{Conclusioni}

Lo studio delle torri difensive della contea di Kaiping dimostra che l'interpretazione e la messa in valore dei diaolou corrisponde alla possibilità che essi siano uno strumento di progettazione e di intervento sul paesaggio del fiume.

I diaolou si costituiscono come un esempio assolutamente contemporaneo e non sono che un'occasione per rimettere in forma un patrimonio più vasto, il territorio fluviale, che necessita di rinnovate attenzioni progettuali, a valle del processo irreversibile di annullamento dei margini della città e di estroflessione fisica di frammenti di città lungo le direttrici dei fiumi.

La necessità di assumere gli elementi naturali quali oggetti di architettura induce ad ampliare il 
dominio dell'architettura della città per approfondire l'architettura della terra, dei fiumi, delle acque.

\section{Note}

${ }^{1}$ Valentina Vacca, in qualità di vincitrice di borsa di ricerca Poliba2China, finanziata dalla Re- gione Puglia nell'ambito delle attività relative al Piano di Azione per la Coesione (PAC), ha svolto un periodo di ricerca presso la School of $\mathrm{Ar}$ chitecture della South China University of Technology di Guangzhou, con la supervisione del Prof. Arch. Changxin Peng.

\section{Bibliography}

Bosselmann, P.C. (2018). Adaptations of the Metropolitan Landscape in Delta Regions, Routledge.

Liang, X.; Yin, J.; Yang, B.; Song, Z. (2017), “The spatial-temporal evolution of Kaiping Diaolou and Villages' defensive functional pattern", Geographical Research, 36, 1, pp. 121-133.

Martí Arís, C. (1994). Le Variazioni Dell'identità: Il Tipo in Architettura, Città Studi Edizioni.

Motta, G.; Ravagnati, C. (2008). Alvei, meandri, isole e altre forme urbane: tecniche di rappresentazione e progetto nei territori fluviali, Franco Angeli.

Motta, G.; Ravagnati, C.; Pizzigoni, A. (2006). L'architettura delle acque e della terra, F. Angeli, Milano.

Tan, J.S. (2007). Kaiping Diaolou and Its Associated Villages: Documenting the Process of Application to the World Heritage List, The University of Hong Kong, Pokfulam, Hong Kong.

Tan, J.S. (2013). The Culture of the Lu Mansion Architecture in China's Kaiping County, 1900-1949.

The State Administration of Cultural Heritage of the People's Republic of China. (2007). "Kaiping Diaolou and Villages. Nomination for World Cultural and Natural Heritage List (Unesco)".

Wang, F. (2016). Geo-Architecture and Landscape in China's Geographic and Historic Context: Volume 4 Symbolism and the Language of Geo-Architecture, Springer Singapore.

Weng, Q. (2007), "A historical perspective of river basin management in the Pearl River Delta of China", Journal of Environmental Management, 85, 4, pp. 1048-1062.

Yin, J.; Tang, X.; Zhang, W.; Liang, X.; Zhu, J. (2019). "Where to Preserve? Evaluating the Integrity Principle for Delineating Protection Scopes of Kaiping Diaolou and Villages", Sustainability, 11, 8, p. 2196.

Zhang, H.; Ma, W.C.; Wang, X.R. (2008). "Rapid Urbanization and Implications for Flood Risk Management in Hinterland of the Pearl River Delta, China: The Foshan Study", Sensors (Basel, Switzerland), 8, 4, pp. 2223-2239.

Zhang, W.; Zhou, H.; Liang, J. (2012). "Comparative research on the categories and typical features of Kaiping diaolou”, Journal of Xi'an University of Architecture \& Technology (Natural Science Edition), 44, 3, pp. 412-419. 
